\title{
New Understanding of the Relevant Role of LINE-1 Retrotransposition in Human Disease and Immune Modulation
}

OPEN ACCESS

Edited by:

Trygve Tollefsbol,

The University of Alabama at Birmingham, United States

Reviewed by:

Sandra Rose Richardson,

The University of Queensland,

Australia

Apiwat Mutirangura,

Chulalongkorn University, Thailand

John LaCava,

The Rockefeller University, United States

*Correspondence: Jinpu Yu

yujinpu@tjmuch.com

Specialty section:

This article was submitted to

Epigenomics and Epigenetics,

a section of the journal

Frontiers in Cell and Developmental

Biology

Received: 15 April 2020

Accepted: 01 July 2020

Published: 07 August 2020

Citation:

Zhang $X$, Zhang $R$ and Yu (2020) New Understanding of the

Relevant Role of LINE-1

Retrotransposition in Human Disease

and Immune Modulation.

Front. Cell Dev. Biol. 8:657.

doi: 10.3389/fcell.2020.00657

\author{
Xiao Zhang ${ }^{1,2}$, Rui Zhang ${ }^{1,2}$ and Jinpu Yu ${ }^{1,2 *}$ \\ ${ }^{1}$ Cancer Molecular Diagnostics Core, Tianjin Medical University Cancer Institute \& Hospital, National Clinical Research \\ Center of Caner, Key Laboratory of Cancer Prevention and Therapy, Key Laboratory of Cancer Immunology and Biotherapy, \\ Tianjin, China, ${ }^{2}$ Tianjin's Clinical Research Center for Cancer, Tianjin, China
}

Long interspersed nuclear element-1 (LINE-1) retrotransposition is a major hallmark of cancer accompanied by global chromosomal instability, genomic instability, and genetic heterogeneity and has become one indicator for the occurrence, development, and poor prognosis of many diseases. LINE-1 also modulates the immune system and affects the immune microenvironment in a variety of ways. Aberrant expression of LINE1 retrotransposon can provide strong stimuli for an innate immune response, activate the immune system, and induce autoimmunity and inflammation. Therefore, inhibition the activity of LINE-1 has become a potential treatment strategy for various diseases. In this review, we discussed the components and regulatory mechanisms involved with LINE-1, its correlations with disease and immunity, and multiple inhibitors of LINE-1, providing a new understanding of LINE-1.

Keywords: retrotransposons, LINE-1, regulatory mechanisms, cancer, immune, inhibitor

\section{INTRODUCTION}

Long interspersed nuclear elements (LINEs) are the only autonomous and active retrotransposons, which include LINE-1, LINE-2, and LINE-3 (Cordaux and Batzer, 2009; de Koning et al., 2011). Also, 5-6\% of LINE-2 and LINE-3 sequences in the human genome are as a truncated molecular fossil (Doxiadis et al., 2012; Ardeljan et al., 2017). LINE-1 retrotransposons are one of the most abundant and effective classes of mobile DNAs that account for $17 \%$ of the human genome (Lander et al., 2001; Hancks and Kazazian, 2016). Full-length LINE-1 is 6.0-7.0 kb and contains a $5^{\prime}$-untranslated region (5'-UTR) (Swergold, 1990), two open reading frames (ORF1 and ORF2), and a $3^{\prime}$-UTR punctuated with a poly-A tract (Babushok and Kazazian, 2007; Beck et al., 2011). Denli et al. (2015) revealed a new open reading frame, ORF0. It is located in the $5^{\prime}$-UTR of the LINE-1 transcript and on the strand opposite of the ORF1 and ORF2 structural genes. Antisense promotor (ASP) can initiate fusion transcripts and regulate ORF0 to enhance LINE-1 mobility (Roman-Gomez et al., 2005; Weber et al., 2010; Criscione et al., 2016).

Both ORFs are required for LINE-1 retrotransposition process. ORF1 encodes an RNA-binding protein named ORF1P that has nucleic acid chaperone activity, and ORF2 encodes a protein named ORF2P that has endonuclease and reverse-transcriptase activities (Mathias et al., 1991; Feng et al., 1996). The first step occurs when RNA polymerase II binds to the $5^{\prime}$-UTR promoter region of LINE-1 and mediates the transcription of full-length mRNA of LINE-1 (Lavie et al., 2004). The LINE-1 mRNA is exported to the cytoplasm where ORF1 and ORF2 are translated and combined to form a ribonucleoprotein (RNP) particle. The RNP is then incorporated into 
the nucleus, and the ORF2P endonuclease in the RNP identifies and cuts specific sequences on the bottom DNA strand at the consensus site $3^{\prime}-\mathrm{AA} / \mathrm{TTTT}-5^{\prime}$. Subsequently, the free $3^{\prime}$ hydroxyl generated at the fracture is utilized by the ORF2P and LINE-1 mRNA in the RNP is used as the template for reverse transcription to produce the complementary DNA of the LINE1 gene (Wei et al., 2001; Hancks and Kazazian, 2016; Wang and Jordan, 2018). The distribution of LINE-1 in the human genome is selective. LINE-1 endonuclease activity and DNA replication determine LINE-1 insertion preference (Flasch et al., 2019). For example, LINE-1 preferentially inserts into nucleosome-depleted DNA primarily as a result of its AT-rich sequences (Sultana et al., 2019). The direction of the DNA replication fork affects LINE1 insertion preference because the cleaved strand is usually the lagging strand template.

LINE-1 elements play a crucial role in the course of species formation and evolution. On one hand, de-repressed LINE-1 functions as a driver of many diseases and even a diagnostic marker for some diseases (Pedersen and Zisoulis, 2016). On the other, it can affect the developmental processes and influence the behavior by generating multiple gene products and causing variable deleterious effects on the structure of the host genome through new insertions, deletions, and recombinations (GarciaPerez et al., 2016). LINE-1 RNA and protein overexpression is related to apoptosis, DNA damage and repair, cellular plasticity, and stress responses and can even promote tumor progression (Morrish et al., 2002, 2007; Belgnaoui et al., 2006; SinibaldiVallebona et al., 2006). DNA damage caused by genomewide or intersperse repetitive sequences hypomethylation can induce inflammatory microenvironment (Lindqvist et al., 2017; Teerawattanapong et al., 2019). Here, we reviewed the correlation between LINE-1 and disease as well as immune system, meanwhile, conducted a new exploration in LINE-1 inhibitors by combining its regulation mechanisms.

Figure 1 shows the relative positions of the $5^{\prime}$ untranslated region ( $5^{\prime}$-UTR); the open reading frames ORF0, ORF1, and ORF2; the $3^{\prime}$ untranslated region ( $3^{\prime}$-UTR); and the Poly A tail. ORF2 encodes endonuclease (EN), reverse transcriptase (RT), and cysteine-rich domain (C). Full-length LINE-1 mRNA was generated using the sense promoter at 5'-UTR. The LINE-1 mRNA is exported to the cytoplasm where ORF1 and ORF2 are translated and combined to form a ribonucleoprotein (RNP) particle. The RNP is then incorporated into the nucleus, and the ORF2P endonuclease in the RNP identifies and cuts specific sequences on the bottom DNA strand at the consensus site $3^{\prime}-\mathrm{AA} / \mathrm{TTTT}-5^{\prime}$. Subsequently, the free $3^{\prime}$ hydroxyl generated at the fracture is utilized by the ORF2P and LINE-1 mRNA in the RNP is used as the template for reverse transcription to produce the complementary DNA of the LINE-1 gene (Richardson et al., 2015; Kazazian and Moran, 2017).

\section{LINE-1 AND DISEASE}

\section{LINE-1 and Cancer}

When LINE-1 retrotransposition is out of control, it can lead to diseases. More than 1,000 articles focusing on LINE-1 and cancer are available in the PubMed archive (Rodic, 2018).

\section{LINE-1 Hypomethylation and Cancer}

The global hypomethylation of the genome promotes chromosomal instability, genomic instability, and genetic heterogeneity because specific changes in DNA methylation can affect a variety of genome sequences, especially the intergenic and intronic regions of the DNA, resulting in chromosome instability and mutations (Wilson et al., 2007). LINE-1 promoter hypomethylation is a biomarker for genome-wide DNA hypomethylation, which is itself a major hallmark of cancer. Thayer et al. (1993) first demonstrated the methylation status of LINE-1 in cancer cells. Since then, LINE-1 hypomethylation of tumors has attracted widespread attention (Thayer et al., 1993). LINE-1 hypomethylation was reported to be associated with poor survival in more than 200 cases of gastric cancer, suggesting its potential as a prognostic biomarker (Shigaki et al., 2013). This phenomenon was also subsequently found in lung cancer, liver cancer, esophageal cancer, prostate cancer, and endometrial cancer (Iwagami et al., 2013; Kawano et al., 2014; Lavasanifar et al., 2019). Ogino et al. (2008) analyzed 643 colon cancer samples from two independent prospective cohorts, demonstrating a linear correlation between LINE1 hypomethylation and aggressive tumor behavior. It has been reported that global DNA hypomethylation promotes aggressive tumor behavior by amplifying oncogenes or through abnormal expression of microRNAs (Baba et al., 2014, 2018). In esophageal cancer with high mortality and poor endoscopic screening sensitivity, LINE-1 hypomethylation can serve as a good diagnostic biomarker, thereby improving 5-year survival (Shah et al., 2013). LINE-1 hypomethylation can also be seen in some precancerous lesions. For example, in colorectal cancer, LINE-1 hypomethylation had no significant difference between adenomas and cancerous tissues, but it was significantly lower in adenomas than in normal tissues (Dawwas, 2014). Therefore, LINE-1 hypomethylation also can be used as an early biomarker for cancer.

However, there was no significant difference in the hypomethylation of LINE-1 between the blood samples of patients with leukemia and those of normal subjects (Barchitta et al., 2014).

\section{LINE-1 Integrations and Cancer}

Many tumor tissues have been found to present a high level of LINE-1 activity that can rapidly increase their copy number through the "copy-and-paste" mechanism (Dunaeva et al., 2018). LINE-1 can be used as cis-regulatory elements to regulate the expression of host genes (Wanichnopparat et al., 2013). Pan-cancer Analysis of Whole Genomes analysis of 2,954 cancer genomes from 38 histological subtypes revealed that aberrant LINE-1 integrations could lead to gene rearrangement (Rodriguez-Martin et al., 2020). LINE-1-mediated rearrangement can trigger oncogene amplification. In breast cancer, Morse and colleagues first proposed that hypomethylation activates LINE1 which can utilize the target primed reverse transcription pathway to insert into the oncogene MYC, causing tumor-specific rearrangement and amplification (Morse et al., 1988). LINE1 was found to induce the amplification of CCND1 oncogene in esophageal tumor by inducing the breakage-fusion-bridge 


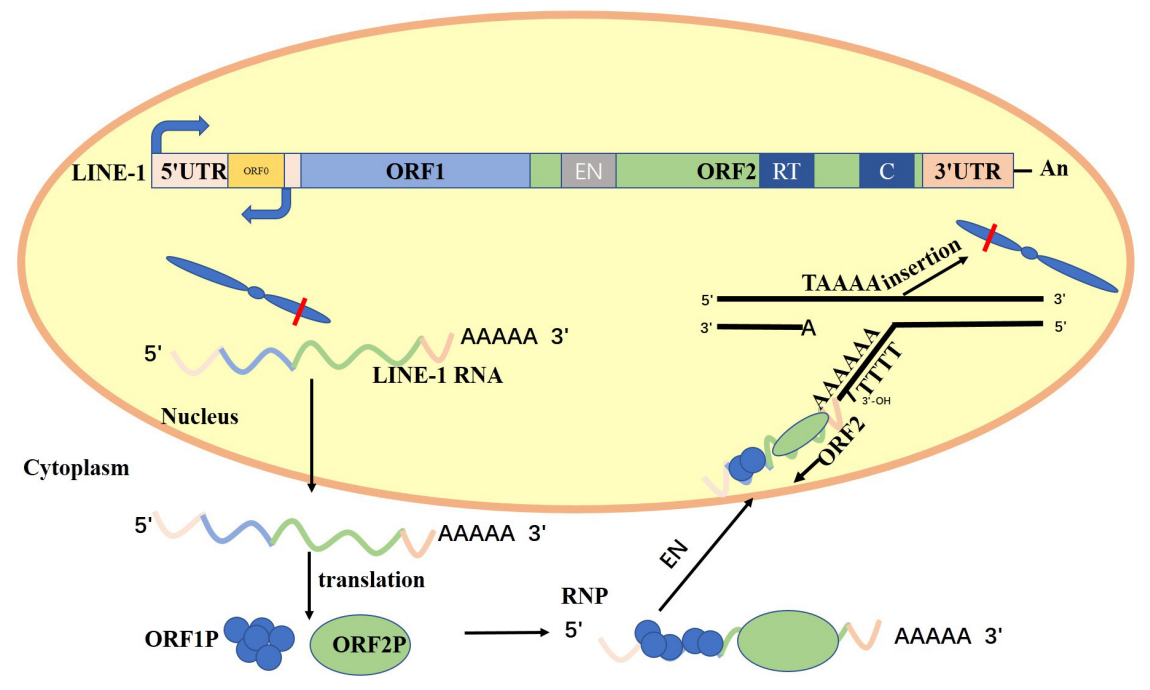

FIGURE 1 | Structure of LINE-1 and LINE-1 retrotransposition cycle.

cycles (Rodriguez-Martin et al., 2020). LINE-1 can mediate the deletion of tumor suppressor genes. It may be through $\mathrm{X}$ inactivation mechanism that LINE-1 mRNA forms facultative heterochromatin in the inactivated region or LINE-1 mRNA and pre-mRNA form RISC complex to degrade complementary mRNA (Allen et al., 2003; Aporntewan et al., 2011). In colon cancer, Miki et al. reported that LINE-1 insertion disrupts the tumor suppressor gene APC, which can lead to gene inactivation (Miki et al., 1992). In lung squamous cell carcinoma, we found that LINE-1 insertion into tumor suppressor gene FGGY promotes cell proliferation and invasion in vitro, and facilitates tumorigenesis in vivo (Zhang et al., 2019).

\section{High Expression of ORF1 and ORF2 of LINE-1 and Cancer}

The activation of LINE-1 increases the translation of ORF1 and ORF2, which are not expressed in normal somatic tissues. ORF1 encodes an RNA-binding protein, and high expression level of ORF1 was proved to be more common in most of the cancers and therefore as a diagnostic marker. In breast cancer, high expression of nuclear ORF1 is associated with distant metastasis and poor prognosis (Harris et al., 2010). In high-grade ovarian carcinoma, the ORF1 level was high and correlated to the loss of TP53 (Rodic et al., 2014). The expression of both the LINE-1 ORF1 and c-Met protein was significantly increased and peaked in early stage in ovarian cancer, suggesting that LINE-1 ORF1 significantly activates c-Met (Ko et al., 2019). In tumor cell experiments, increased mRNA and protein expression of LINE1ORF1 can result in significant enhancement in cell proliferation and colony formation (Tang et al., 2018). It is worth noting that the expression of ORF1 was heterogeneous and had histological specificity. Cancers originating in the endometrium, such as biliary tract, esophagus, bladder, head and neck, lung, and colon, exhibit ORF1 overexpression, whereas other cancers, such as renal, liver, and cervical cancer, show little expression of ORF1
(Ardeljan et al., 2017). Recent studies have shown that an ELISA method to measure ORF1 in serum can be better in prostate cancer detection (Hosseinnejad et al., 2018).

ORF2 encodes a protein with reverse transcriptase and endonuclease activities. High expression of endonuclease induces double-strand DNA breakage that can aggravate DNA damage repair and increase genomic instability (Kines et al., 2014). Reverse transcriptase activation can promote cell proliferation and differentiation and also alter the non-coding RNA transcription spectrum and other epigenetic phenotypes, resulting in alterations in cell regulatory networks, tumor development, and other important pathological processes (Rodic and Burns, 2013; Burns, 2017; Christian et al., 2017). ORF2 can express early in the tumorigenesis process, as it can be detected by a highly specific monoclonal antibody (mAb chA1-L1) in both transitional colon mucosa and prostate intraepithelial neoplasias (De Luca et al., 2016). However, studies have shown that chA1L1 recognizes both ORF2p and the transcriptional regulator BCLAF1, so it is not specific (Briggs et al., 2019). But recently, tumor proteome profiling studies based on mass spectrometry have shown that ORF2p was difficult to be detected, and after affinity capture of ORF1p, ORF2p has not been detected in stem cell LINE-1 proteome analysis (Vuong et al., 2019; Ardeljan et al., 2020). Therefore, the detection and application of ORF2 in tumors are still worth exploring.

\section{LINE-1 and Metabolic Disorders}

New research has shown that LINE-1 is also associated with blood sugar and lipid levels (Turcot et al., 2012). LINE-1 methylation is associated with type 2 diabetes mellitus (T2DM). Studies showed that, compared with hypermethylation, LINE-1 hypomethylation was associated with a higher risk of worsening metabolic status, independent of other classic risk factors (Martin-Nunez et al., 2014). This discovery highlights the potential role for LINE-1 DNA methylation as a predictor of the risk of T2DM or 
other related metabolic disorders. LINE-1 DNA methylation is associated with increased LDL cholesterol and decreased HDL cholesterol levels, and these metabolic changes increase the risk of cardiovascular disease (Pearce et al., 2012). LINE-1 DNA methylation is also associated with many blood-based metabolic biomarkers. In fetal neural tissue with neural tube defects, it was found that the low methylation level of LINE-1 was associated with the significant reduction of vitamin $B_{12}$ in maternal plasma, as well as lower folate levels and increased concentrations of homocysteine (Wang et al., 2010). Folic acid and other B vitamins play an important role in the biosynthesis of new purines and pyrimidines. Therefore, the methylation status of LINE- 1 can be a predictor of some metabolic diseases. Current studies have shown that LINE-1 can also regulate metabolism by inserting metabolic genes. It was reported that LINE-1 insertions in the FGGY gene can upregulate cytochrome $\mathrm{P} 450$, arachidonic acid metabolism, and glycerolipid metabolism. These metabolic disorders can lead to the occurrence of a variety of diseases and poor prognosis (Zhang et al., 2019).

\section{LINE-1 and Neurological Disorders}

LINE-1 can affect the developing brain at different stages of health and disease (Suarez et al., 2018). Ataxia telangiectasia (AT) is a progressive neurodegenerative disease caused by ataxia telangiectasia mutated (ATM) gene mutation. In 2011, researchers found that in nasopharyngeal carcinomas with ATM deficiency, LINE-1 retrotransposition increased, and ORF2 copy number increased in AT neurons, thus verifying the correlation between LINE-1 retrotransposition and ATM deficiency (Coufal et al., 2011). High expression of LINE-1 was found in Rett syndrome caused by mutation of methyl CpG binding protein 2 (MeCP2) in the $\mathrm{X}$-linked gene, which was caused by the inclusion of LINE-1 $5^{\prime}$-UTR sequence in the MeCP2 target, leading to methylation-dependent repression (Muotri et al., 2010). LINE-1 is involved in the aging process. In patients with frontotemporal lobe degeneration, LINE-1 transcripts were found to be elevated ( $\mathrm{Li}$ et al., 2012). LINE-1 hypomethylation has been observed in most psychiatric studies. Increased copy numbers of LINE-1 as a result of LINE-1 hypomethylation were also found in patients with schizophrenia, bipolar disorder, and major depressive disorder (Liu et al., 2016; Li et al., 2018). The link between LINE-1 methylation levels and Alzheimer's disease is still being studied.

\section{LINE-1 and Genetic Disorders}

LINE-1 is reported to be related to chromosome disorders. The first observation of LINE-1 insertion was in 1988, when Kazazian et al. observed a new exon of F8 LINE-1 insertion in the X-linked gene, which is a gene encoding coagulation factor VIII in a patient with hemophilia A (Kazazian et al., 1988). Then, a LINE-1 insertion was found in the CHM gene of a patient diagnosed with choroideremia. The reverse integration of a LINE-1 element into exon 6 resulted in aberrant splicing of the CHM mRNA (van den Hurk et al., 2003). Furthermore, LINE-1 can also promote mobilization of other RNAs in trans, Alu, and SVA, which can be trans-mobilized, leading to gene insertions (Kemp and Longworth, 2015). Retrotransposon insertions were found to account for up to $0.4 \%$ of all NF1 mutations (Wimmer et al., 2011). Neurofibromatosis type I is an autosomal dominant disorder caused by NF1 gene mutations (Messiaen et al., 2011). Alu insertion is located 44 bp upstream of NF1 exon 41, causing the exon 41 to skip and change the open reading frame (Payer and Burns, 2019). Only two cases were thought to be a result of independent SVA insertion in SUZ12P accompanied by $867-\mathrm{kb}$ and 1-Mb deletions that encompassed the NF1 gene (Vogt et al., 2014). In autosomal recessive genetic disease, such as Fanconi anemia caused by SLX $4^{\text {FANCP }}$ deficiency and Aicardi-Goutieres syndrome (AGS) of three-prime repair exonuclease 1 mutations, LINE-1 expression was upregulated and pro-inflammatory cytokines were produced through the cGASSTING pathway (Brégnard et al., 2016; Suarez et al., 2018).

\section{LINE-1 AND IMMUNE REGULATION OF DISEASE}

\section{LINE-1 and Autoimmune Disease}

Hypomethylated and highly expressed LINE-1 has been found in autoimmune diseases such as systemic lupus erythematosus (SLE), Sjögren's syndrome (SS), and psoriasis (Schulz et al., 2006; Yooyongsatit et al., 2015; Mavragani et al., 2016). LINE-1 RNA is characterized by viral RNA and exists as RNP particles, which can be recognized by RNA sensors and activate innate immune responses (Mavragani et al., 2016). Cell studies demonstrated that LINE- 1 activates the production of IFN $\beta$ by RNA pathway (Zhao et al., 2018). When LINE-1 retrotransposition was inhibited by RT inhibitors, significant reductions were observed in IFN $\alpha$, IFN $\beta$, and IFN $\gamma$ mRNA levels (Brégnard et al., 2016). LINE-1 transcripts and $\mathrm{p} 40$ protein (a $40-\mathrm{kDa} \mathrm{RNA}$ binding protein) that LINE-1 encodes have been detected in SLE and SS patients. It has been demonstrated that LINE-1 can induce the production of IFN-I in vitro by TLR-dependent and TLR-independent pathways (Mavragani et al., 2016). In MRL autoimmune lymphoproliferative syndrome, LINE-1 ORF2 encoding an RT and its products are associated with an MHC class I molecule on the cell membrane (Benihoud et al., 2002). In Fanconi anemia and AGS, LINE-1 was found to be associated with the activation of the autoimmune system. LINE-1 also regulates immunity by acting as a cis-regulatory element through the mechanism of LINE-1 mRNA and pre-mRNA forming RISC complex to degrade the complementary mRNA (Wanichnopparat et al., 2013).

\section{LINE-1 and Tumor Immunity}

In 112 TCGA cancer samples, the scientists measured the transcriptional activity of 1789 pathways and found that 49 of 176 immune pathways were significantly negatively correlated with LINE-1 (Jung et al., 2018). LINE-1 is inversely correlated with the expression of immunologic response genes. Less LINE1 activity was found in tumors with high immune activity. In esophageal cancer tissues, scientists found that the LINE-1 methylation level in tumors was significantly positively associated with the peritumoral lymphocytic reaction (Kosumi et al., 2019). The activities of regulatory T cells and PD1 signaling as reported in cancer immune evasion and chronic inflammatory conditions 
also have negative correlations with LINE-1. It is reported that the negative correlation between LINE-1 and immune activity may be caused by the destruction of LINE-1 inhibition, but the specific mechanism is still unclear. LINE-1 may also mediate immune tolerance, which may change from immune stimulation mode to immunosuppression mode through continuous IFN signaling or directly affect lymphocyte signaling.

\section{LINE-1 and Metabolism-Induced Immunity}

LINE-1 is also associated with blood sugar and lipid levels. Abnormal glucose and lipid metabolism can lead to metabolic reprogramming in tumor cells. The most classic metabolism of tumor is Warburg effect, where a large amount of glucose is absorbed to fulfill the need for proliferation and produce lactic acid (Lunt and Vander Heiden, 2011). The acidic microenvironment caused by lactic acid leads to impaired T-cell activation and proliferation, prevents NK cell activation, stabilizes HIF1 $\alpha$ to stimulate the polarization of antiinflammatory M2 macrophages, and inhibits the production of IFN- $\gamma$ in tumor-infiltrating T cells (Husain et al., 2013; Colegio et al., 2014; Brand et al., 2016). Abnormal lipid metabolism in tumor cells also can lead to local immunosuppression in the microenvironment (Hao et al., 2019). LINE-1 can affect local immune homeostasis by inserting elements into metabolismrelated genes. FGGY is known to encode a protein that phosphorylates carbohydrates and is associated with obesity and sporadic amyotrophic lateral sclerosis (Zhang et al., 2011). LINE1 retrotransposons suppress FGGY, leading to lipid metabolism disturbance and diet-induced obesity in mice (Taylor et al., 2018). Lung squamous cell carcinoma patients with L1-FGGY ${ }^{+}$ tissue have a poor prognosis, have low levels of $\mathrm{CD}^{+} \mathrm{T}$ cells, and have high levels of $\mathrm{CD}^{+} 8^{+}$macrophages and $\mathrm{CD} 33^{+}$ myeloid-derived cells (Zhang et al., 2019). L1-FGGY ${ }^{+}$also regulates the abnormal transcription of cytokines related to the immunosuppressive micromilieu.

\section{LINE-1 INHIBITION}

The correlation between LINE-1 and disease as well as immunity was analyzed (Figure 2). The life cycle of LINE-1 provides a plethora of ways to target and inhibit LINE-1 expression
(Banuelos-Sanchez et al., 2019). The inhibition of LINE-1 has become a treatment strategy for some diseases.

\section{Targeting LINE-1 Methylation}

Full-length LINE-1 transcription is driven by a CpG dinucleotide-rich internal promoter. Hypomethylation of LINE1 causes the activation of LINE-1, which causes retroelement transposition and chromosomal alteration (Saito et al., 2010). The hypomethylation of LINE-1 has become an important factor in the occurrence and development of diseases, so maintaining the state of LINE-1 methylation has become a key method for the treatment of diseases. Soy isoflavone supplementation can regulate the level of LINE-1 methylation in head and neck squamous cell carcinoma (HNSCC). In a clinical trial of 39 patients with HNSCC who took a soy isoflavone supplement (300 mg/day) orally for 3 weeks before surgery, a positive correlation was found between LINE-1 methylation level and daily isoflavone intake (Rozek et al., 2019). Some cell-based studies and clinical data have shown that LINE-1 dysregulation is associated with tumor drug resistance (Zhu et al., 2015; Lavasanifar et al., 2019). It was found in breast cancer cells treated with paclitaxel that DNMT3a, a member of the DNA methyltransferase family, could enhance the methylation level in the gene by binding to the inner region of the LINE-1 gene, and then upregulate the expression level of LINE-1. Downregulating the expression of DNMT3a can effectively inhibit the expression of LINE-1 (Wang et al., 2020). LINE-1 retrotransposon silenced also through histone modifications. Histone demethylase KDM4B may enhance the LINE-1 retrotransposition efficacy, whereas depletion of KDM4B reduced it in breast cancer (Xiang et al., 2019). Elevated LINE-1 expression was found in PC9 drug-tolerant persister (DTP) cancer cells treated with the EGFR inhibitor erlotinib. HDAC inhibitors can suppress LINE-1 in DTP cancer cells (Guler et al., 2017). Currently, DNA methyltransferase inhibitors and histone deacetylase inhibitors have entered clinical trials (Gaillard et al., 2019).

\section{Targeting RT Activity}

LINE-1 elements harbor ORF1 and ORF2, which has reverse transcriptase (RT) activity, and RT inhibition may be a novel, non-cytotoxic anticancer therapy strategy (Sciamanna et al., 2018). RT is a key player in retrotransposition and functions by transcribing LINE-1 mRNA or other RNAs to

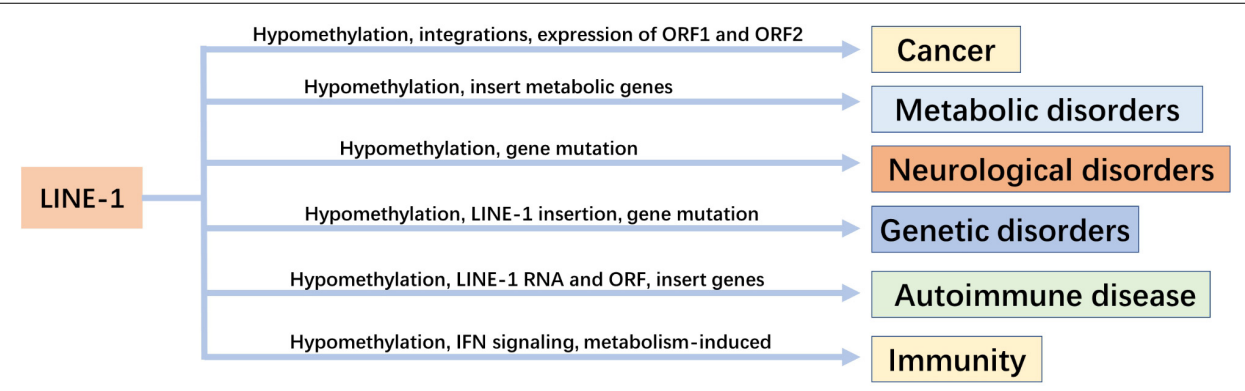

FIGURE 2 | The relationship between LINE-1 and diseases and their regulatory mechanisms. 
cDNA at the integration sites (Khalid et al., 2018). Specific reverse transcription inhibitors, including nevirapine (NVR) and efavirenz (EFV), which target the HIV-1-encoded RT and are currently used in AIDS therapy, reduce cell proliferation and promotes differentiation of a variety of cancer cell lines of unrelated histological origin (Mangiacasale et al., 2003; Landriscina et al., 2005; Sciamanna et al., 2005). In vivo assays using murine models inoculated with various human cancer cell lines revealed that daily treatment of animals with EFV significantly delayed the progression of tumors (Oricchio et al., 2007). NVR and EFV dramatically countered L1-FGGY abundance, inhibited tumor growth, attenuated metabolism dysfunction, and improved the local immune evasion in lung squamous cell carcinomas (Zhang et al., 2019). EFV has recently undergone a phase II clinical trial in patients with metastatic prostate cancer (Houédé et al., 2014). Another RT inhibitor, F2-DABOs, has shown anti-proliferative activity in nude mice, helping to promote cell differentiation and inhibit tumor growth (Sbardella et al., 2011). Later, the nucleoside reverse transcription inhibitor abacavir was also shown to inhibit cell growth, migration, and invasion (Carlini et al., 2010). Capsaicin is the main chemical component of Asiasari radix and Capsicum annuum, as well as the major component of a Chinese traditional herbal medicine, Sho-seiryu-to (Friedman et al., 2018). Capsaicin suppresses LINE-1 by inhibiting the RT activity of LINE-1 ORF2P, which is the LINE-1-encoded RT responsible for LINE-1 activity (Nishikawa et al., 2018). A recent study revealed that azidothymidine (AZT) inhibits the RT activity of ORF2P in a fetal oocyte attrition model. Experiments showed that AZT-treated oocytes have a reduction of LINE-1 ORF1 ssDNA compared with untreated oocytes (Tharp et al., 2020). It is important to note that RT inhibitors do not eliminate the tumor but only control its progression. Therefore, in addition to the anti-AIDS drugs approved by the FDA, the combination of Chinese and western medicine can be regarded as an emerging treatment.

\section{Combined Immunotherapy}

Recent studies suggest that LINE-1 hypomethylation may be a positive indicator of immunotherapy. DNA methyltransferase (DNMT) is an important epigenetic molecule that catalyzed DNA methylation and can induce the development of various tumors. Downregulating the expression of DNMT can effectively inhibit the expression of LINE-1 (Wang et al., 2020). So DNA methyltransferase inhibitors (DNMTis) play an important role in the anti-tumor process. DNMTI can improve tumor immunogenicity, promote $\mathrm{NK}$ cells and $\mathrm{CD}^{+} \mathrm{T}$ cells to play a cell-mediated cytotoxic role, and promote immune response to participate in antigen commission by regulating immunosuppressive cells (Chiappinelli et al., 2015). DNMTi can enhance the expression of cancer-testis (CT) antigen, making the tumor more susceptible to CT antigen vaccine. The combination of decitabine, a DNA methyltransferase inhibitor, and cancer-testis/cancer-germline antigen NY-ESO-1 vaccine has a good therapeutic effect in the primary treatment of human recurrent epithelial ovarian cancer (Odunsi et al., 2014). A clinical trial has shown that combination therapy with carboplatin and anti-programmed death-1 has a good therapeutic effect in lung cancer because carboplatin can induce LINE-1 expression (Langer et al., 2016). Therefore, LINE- 1 can be used as a target of combined immunotherapy in tumor therapy.

\section{Other Inhibitors}

Recently, a number of other regulatory approaches have been reported. In somatic cells, microRNAs (miRNAs or miRs) also regulate the activity of LINE-1 (Idica et al., 2017). MiR-128 regulates LINE-1 activity in somatic cells by targeting the nuclear import factor transportin-1 (TNPO1) 3'-UTR, which mediates nuclear import and requires RanGTP for cargo delivery into the nucleus (Twyffels et al., 2014). MiR-128 inhibits the expression of TNPO1 mRNA and protein, and TNPO1 deficiency suppresses LINE-1 mobilization by inhibiting nuclear import of LINE1-RNP (Idica et al., 2017). MiR-128 also guides the miRNAinduced silencing complex to bind directly to a target site residing in the ORF2 RNA of LINE-1 (Hamdorf et al., 2015). At present, a novel target of miR-128 has been identified as heterogeneous nuclear ribonucleoprotein A1 (hnRNPA1), which is required for LINE-1 retrotransposition (Goodier et al., 2013; Fung et al., 2019). MiR-128 represses hnRNPA1 mRNA and protein by targeting the CDS of hnRNPA1, which interacts with LINE-1 ORF1p via RNA bridge to promote LINE-1 mobilization (Goodier et al., 2013). This interaction results in translational repression of the LINE-1 retrotransposition, thereby reducing the risk of LINE-1-mediated mutagenesis (Pedersen and Zisoulis, 2016). Therefore, microRNAs can be a target for LINE-1 inhibition.

Aryl hydrocarbon receptor (AHR) is a ligand-activated transcription factor that activates LINE-1 expression (Teneng et al., 2007). AHR is overexpressed in breast and thyroid cancers, suggesting that these tumors also overexpress LINE-1 (Powell et al., 2013). Lai et al. found that biseugenol, a novel AHR inhibitor, impeded cancer growth and inhibited EMT in gastric cancer cells (Lai et al., 2014). These findings suggest that targeting AHR with small molecule inhibitors may be a novel therapeutic approach. ORF1P phosphorylation by protein kinase $\mathrm{A}$ is also required for LINE-1. Kinase inhibitors specifically designed to target LINE-1 ORF1P phosphorylation may be associated with inhibition of LINE-1 (Bojang et al., 2013). Therefore, there is room for drug development research focusing on targeting and inhibiting LINE-1 ORF1P phosphorylation.

\section{CONCLUSION}

The activation of LINE-1 retrotransposon is associated with a variety of human diseases and is involved in the occurrence and progression of disease through retrotransposition-dependent and retrotransposition-independent mechanisms. Currently, it has even become a marker of tumorigenesis and prognosis and is related to immune regulation. The effective inhibition of LINE-1 activation has become a treatment for some diseases. The inhibition of LINE-1 in animal experiments can inhibit 
the occurrence and development of tumors, so the clinical application of LINE-1 inhibitors is imminent. In addition to exploring some known inhibitors, other mechanisms of LINE1 inhibition should also be explored. We summarized the relationship between LINE-1 and disease-related immunity, and proposed that LINE-1 may affect the immune status of the body by regulating metabolism, leading to poor prognosis. Metabolic substances can affect the immune microenvironment, for example, lactic acid can lead to immunosuppressive microenvironment, leading to poor prognosis of tumors. The dysregulation of LINE-1 can lead to the disorder of glucose and lipid metabolism, and the inhibition of glucose and lipid metabolism may reverse the disease progression caused by LINE-1. Now the anti-tumor effect of regulating the body's metabolism has entered clinical trials, such as the significant effect of metformin in the treatment of tumors. Therefore, the metabolic status of diseases caused by LINE-1 can be checked. Metabolic therapy combined with LINE-1 inhibitors may inhibit

\section{REFERENCES}

Allen, E., Horvath, S., Tong, F., Kraft, P., Spiteri, E., Riggs, A. D., et al. (2003). High concentrations of long interspersed nuclear element sequence distinguish monoallelically expressed genes. Proc. Natl. Acad. Sci. U.S.A. 100, 9940-9945. doi: 10.1073/pnas.1737401100

Aporntewan, C., Phokaew, C., Piriyapongsa, J., Ngamphiw, C., Ittiwut, C., Tongsima, S., et al. (2011). Hypomethylation of intragenic LINE-1 represses transcription in cancer cells through AGO2. PLoS One 6:e17934. doi: 10.1371/ journal.pone.0017934

Ardeljan, D., Taylor, M. S., Ting, D. T., and Burns, K. H. (2017). The human long interspersed element-1 retrotransposon: an emerging biomarker of neoplasia. Clin. Chem. 63, 816-822. doi: 10.1373/clinchem.2016.257444

Ardeljan, D., Wang, X., Oghbaie, M., Taylor, M. S., Husband, D., Deshpande, V., et al. (2020). LINE-1 ORF2p expression is nearly imperceptible in human cancers. Mob. DNA 11:1. doi: 10.1186/s13100-019-0191-2

Baba, Y., Watanabe, M., Murata, A., Shigaki, H., Miyake, K., Ishimoto, T., et al. (2014). LINE-1 hypomethylation, DNA copy number alterations, and CDK6 amplification in esophageal squamous cell carcinoma. Clin. Cancer Res. 20, 1114-1124. doi: 10.1158/1078-0432.CCR-13-1645

Baba, Y., Yagi, T., Sawayama, H., Hiyoshi, Y., Ishimoto, T., Iwatsuki, M., et al. (2018). Long interspersed element-1 methylation level as a prognostic biomarker in gastrointestinal cancers. Digestion 97, 26-30. doi: 10.1159/ 000484104

Babushok, D. V., and Kazazian, H. H. Jr. (2007). Progress in understanding the biology of the human mutagen LINE-1. Hum. Mutat. 28, 527-539. doi: 10. 1002/humu.20486

Banuelos-Sanchez, G., Sanchez, L., Benitez-Guijarro, M., Sanchez-Carnerero, V., Salvador-Palomeque, C., Tristan-Ramos, P., et al. (2019). Synthesis and characterization of specific reverse transcriptase inhibitors for mammalian LINE-1 retrotransposons. Cell Chem. Biol. 26, 1095-1109.e14. doi: 10.1016/j. chembiol.2019.04.010

Barchitta, M., Quattrocchi, A., Maugeri, A., Vinciguerra, M., and Agodi, A. (2014). LINE-1 hypomethylation in blood and tissue samples as an epigenetic marker for cancer risk: a systematic review and meta-analysis. PLoS One 9:e109478. doi: 10.1371/journal.pone.0109478

Beck, C. R., Garcia-Perez, J. L., Badge, R. M., and Moran, J. V. (2011). LINE-1 elements in structural variation and disease. Annu. Rev. Genom. Hum. Genet. 12, 187-215. doi: 10.1146/annurev-genom-082509-141802

Belgnaoui, S. M., Gosden, R. G., Semmes, O. J., and Haoudi, A. (2006). Human LINE-1 retrotransposon induces DNA damage and apoptosis in cancer cells. Cancer Cell Int. 6:13. doi: 10.1186/1475-2867-6-13

Benihoud, K., Bonardelle, D., Soual-Hoebeke, E., Durand-Gasselin, I., Emilie, D., Kiger, N., et al. (2002). Unusual expression of LINE-1 transposable element in the progression of LINE-1 and may improve the immune microenvironment to achieve the optimal therapeutic effect.

\section{AUTHOR CONTRIBUTIONS}

XZ wrote the article. RZ and JY reviewed and revised the article. All authors contributed to the article and approved the submitted version.

\section{FUNDING}

This work was supported by the National Natural Science Foundation of China (Grant Nos. 81702280 and 81872143), National Science and Technology Support Program of China (Grant No. 2018ZX09201015), and Project of Science and Technology of Tianjin (Grant No. 18JCQNJC82700).

the MRL autoimmune lymphoproliferative syndrome-prone strain. Oncogene 21, 5593-5600. doi: 10.1038/sj.onc.1205730

Bojang, P. Jr., Roberts, R. A., Anderton, M. J., and Ramos, K. S. (2013). Reprogramming of the HepG2 genome by long interspersed nuclear element-1. Mol. Oncol. 7, 812-825. doi: 10.1016/j.molonc.2013.04.003

Brand, A., Singer, K., Koehl, G. E., Kolitzus, M., Schoenhammer, G., Thiel, A., et al. (2016). LDHA-associated lactic acid production blunts tumor immunosurveillance by T and NK cells. Cell Metab. 24, 657-671. doi: 10.1016/ j.cmet.2016.08.011

Brégnard, C., Guerra, J., Déjardin, S., Passalacqua, F., Benkirane, M., and Laguette, N. (2016). Upregulated LINE-1 activity in the fanconi anemia cancer susceptibility syndrome leads to spontaneous pro-inflammatory cytokine production. EBioMedicine 8, 184-194. doi: 10.1016/j.ebiom.2016.05.005

Briggs, E. M., Spadafora, C., and Logan, S. K. (2019). A re-evaluation of LINE1 ORF2 expression in LNCaP prostate cancer cells. Mob. DNA 10:51. doi: 10.1186/s13100-019-0196-x

Burns, K. H. (2017). Transposable elements in cancer. Nat. Rev. Cancer 17, 415-424. doi: 10.1038/nrc.2017.35

Carlini, F., Ridolfi, B., Molinari, A., Parisi, C., Bozzuto, G., Toccacieli, L., et al. (2010). The reverse transcription inhibitor abacavir shows anticancer activity in prostate cancer cell lines. PLoS One 5:e14221. doi: 10.1371/journal.pone. 0014221

Chiappinelli, K. B., Strissel, P. L., Desrichard, A., Li, H., Henke, C., Akman, B., et al. (2015). Inhibiting DNA methylation causes an interferon response in cancer via dsRNA including endogenous retroviruses. Cell 162, 974-986. doi: 10.1016/j.cell.2015.07.011

Christian, C. M., Sokolowski, M., deHaro, D., Kines, K. J., and Belancio, V. P. (2017). Involvement of conserved amino acids in the C-terminal region of LINE-1 ORF2p in retrotransposition. Genetics 205, 1139-1149. doi: 10.1534/ genetics.116.191403

Colegio, O. R., Chu, N. Q., Szabo, A. L., Chu, T., Rhebergen, A. M., Jairam, V., et al. (2014). Functional polarization of tumour-associated macrophages by tumour-derived lactic acid. Nature 513, 559-563. doi: 10.1038/nature 13490

Cordaux, R., and Batzer, M. A. (2009). The impact of retrotransposons on human genome evolution. Nat. Rev. Genet. 10, 691-703. doi: 10.1038/nrg 2640

Coufal, N. G., Garcia-Perez, J. L., Peng, G. E., Marchetto, M. C., Muotri, A. R., $\mathrm{Mu}, \mathrm{Y}$., et al. (2011). Ataxia telangiectasia mutated (ATM) modulates long interspersed element-1 (L1) retrotransposition in human neural stem cells. Proc. Natl. Acad. Sci. U.S.A. 108, 20382-20387. doi: 10.1073/pnas.1100273108

Criscione, S. W., Theodosakis, N., Micevic, G., Cornish, T. C., Burns, K. H., Neretti, N., et al. (2016). Genome-wide characterization of human L1 antisense promoter-driven transcripts. BMC Genomics 17:463. doi: 10.1186/s12864-0162800-5 
Dawwas, M. F. (2014). Adenoma detection rate and risk of colorectal cancer and death. N. Engl. J. Med. 370, 2539-2540. doi: 10.1056/NEJMc1405329

de Koning, A. P., Gu, W., Castoe, T. A., Batzer, M. A., and Pollock, D. D. (2011). Repetitive elements may comprise over two-thirds of the human genome. PLoS Genet. 7:e1002384. doi: 10.1371/journal.pgen.1002384

De Luca, C., Guadagni, F., Sinibaldi-Vallebona, P., Sentinelli, S., Gallucci, M., Hoffmann, A., et al. (2016). Enhanced expression of LINE-1-encoded ORF2 protein in early stages of colon and prostate transformation. Oncotarget 7 , 4048-4061. doi: 10.18632/oncotarget.6767

Denli, A. M., Narvaiza, I., Kerman, B. E., Pena, M., Benner, C., Marchetto, M. C. N., et al. (2015). Primate-specific ORF0 contributes to retrotransposon-mediated diversity. Cell 163, 583-593. doi: 10.1016/j.cell.2015.09.025

Doxiadis, G. G., Hoof, I., de Groot, N., and Bontrop, R. E. (2012). Evolution of HLA-DRB genes. Mol. Biol. Evol. 29, 3843-3853. doi: 10.1093/molbev/mss186

Dunaeva, M., Derksen, M., and Pruijn, G. J. M. (2018). LINE-1 hypermethylation in serum cell-free DNA of relapsing remitting multiple sclerosis patients. Mol. Neurobiol. 55, 4681-4688. doi: 10.1007/s12035-017-0679-z

Feng, Q., Moran, J. V., Kazazian, H. H. Jr., and Boeke, J. D. (1996). Human L1 retrotransposon encodes a conserved endonuclease required for retrotransposition. Cell 87, 905-916. doi: 10.1016/S0092-8674(00)81997-2

Flasch, D. A., Macia, Á, Sánchez, L., Ljungman, M., Heras, S. R., García-Pérez, J. L., et al. (2019). Genome-wide de novo L1 retrotransposition connects endonuclease activity with replication. Cell 177, 837-851.e28. doi: 10.1016/j. cell.2019.02.050

Friedman, J. R., Nolan, N. A., Brown, K. C., Miles, S. L., Akers, A. T., Colclough, K. W., et al. (2018). Anticancer activity of natural and synthetic capsaicin analogs. J. Pharmacol. Exp. Ther. 364, 462-473. doi: 10.1124/jpet.117.243691

Fung, L., Guzman, H., Sevrioukov, E., Idica, A., Park, E., Bochnakian, A., et al. (2019). miR-128 restriction of LINE-1 (L1) retrotransposition is dependent on targeting hnRNPA1 mRNA. Int. J. Mol. Sci. 20, 1955 . doi: 10.3390/ ijms20081955

Gaillard, S. L., Zahurak, M., Sharma, A., Durham, J. N., Reiss, K. A., SartoriusMergenthaler, S., et al. (2019). A phase 1 trial of the oral DNA methyltransferase inhibitor CC-486 and the histone deacetylase inhibitor romidepsin in advanced solid tumors. Cancer 125, 2837-2845. doi: 10.1002/cncr.32138

Garcia-Perez, J. L., Widmann, T. J., and Adams, I. R. (2016). The impact of transposable elements on mammalian development. Development (Cambridge, England) 143, 4101-4114. doi: 10.1242/dev.132639

Goodier, J. L., Cheung, L. E., and Kazazian, H. H. Jr. (2013). Mapping the LINE1 ORF1 protein interactome reveals associated inhibitors of human retrotransposition. Nucleic Acids Res. 41, 7401-7419. doi: 10.1093/nar/ gkt512

Guler, G. D., Tindell, C. A., Pitti, R., Wilson, C., Nichols, K., KaiWai Cheung, T., et al. (2017). Repression of stress-induced LINE-1 expression protects cancer cell subpopulations from lethal drug exposure. Cancer Cell 32, 221-237.e13. doi: 10.1016/j.ccell.2017.07.002

Hamdorf, M., Idica, A., Zisoulis, D. G., Gamelin, L., Martin, C., Sanders, K. J., et al. (2015). miR-128 represses L1 retrotransposition by binding directly to L1 RNA. Nat. Struct. Mol. Biol. 22, 824-831. doi: 10.1038/nsmb.3090

Hancks, D. C., and Kazazian, H. H. Jr. (2016). Roles for retrotransposon insertions in human disease. Mob. DNA 7:9. doi: 10.1186/s13100-0160065-9

Hao, Y., Li, D., Xu, Y., Ouyang, J., Wang, Y., Zhang, Y., et al. (2019). Investigation of lipid metabolism dysregulation and the effects on immune microenvironments in pan-cancer using multiple omics data. BMC Bioinformatics 20:195. doi: 10. 1186/s12859-019-2734-4

Harris, C. R., Normart, R., Yang, Q., Stevenson, E., Haffty, B. G., Ganesan, S., et al. (2010). Association of nuclear localization of a long interspersed nuclear element-1 protein in breast tumors with poor prognostic outcomes. Genes Cancer 1, 115-124. doi: 10.1177/1947601909360812

Hosseinnejad, K., Yin, T., Gaskins, J. T., Bailen, J. L., and Jortani, S. A. (2018). Discovery of the long interspersed nuclear element-1 activation product [Open Reading Frame-1 (ORF1) protein] in human blood. Clin. Chim. Acta 487, 228-232. doi: 10.1016/j.cca.2018.09.040

Houédé, N., Pulido, M., Mourey, L., Joly, F., Ferrero, J.-M., Bellera, C., et al. (2014). A phase II trial evaluating the efficacy and safety of efavirenz in metastatic castration-resistant prostate cancer. Oncologist 19, 1227-1228. doi: 10.1634/ theoncologist.2014-0345
Husain, Z., Huang, Y., Seth, P., and Sukhatme, V. P. (2013). Tumor-derived lactate modifies antitumor immune response: effect on myeloid-derived suppressor cells and NK cells. J. Immunol. 191, 1486-1495. doi: 10.4049/jimmunol. 1202702

Idica, A., Sevrioukov, E. A., Zisoulis, D. G., Hamdorf, M., Daugaard, I., Kadandale, P., et al. (2017). MicroRNA miR-128 represses LINE-1 (L1) retrotransposition by down-regulating the nuclear import factor TNPO1. J. Biol. Chem. 292, 20494-20508. doi: 10.1074/jbc.M117.807677

Iwagami, S., Baba, Y., Watanabe, M., Shigaki, H., Miyake, K., Ishimoto, T., et al. (2013). LINE-1 hypomethylation is associated with a poor prognosis among patients with curatively resected esophageal squamous cell carcinoma. Ann. Surg. 257, 449-455. doi: 10.1097/SLA.0b013e31826d8602

Jung, H., Choi, J. K., and Lee, E. A. (2018). Immune signatures correlate with L1 retrotransposition in gastrointestinal cancers. Genome Res. 28, 1136-1146. doi: $10.1101 /$ gr.231837.117

Kawano, H., Saeki, H., Kitao, H., Tsuda, Y., Otsu, H., Ando, K., et al. (2014). Chromosomal instability associated with global DNA hypomethylation is associated with the initiation and progression of esophageal squamous cell carcinoma. Ann. Surg. Oncol. 21(Suppl. 4), S696-S702. doi: 10.1245/s10434014-3818-z

Kazazian, H. H. Jr., and Moran, J. V. (2017). Mobile DNA in health and disease. N Engl. J. Med. 377, 361-370. doi: 10.1056/NEJMra1510092

Kazazian, H. H. Jr., Wong, C., Youssoufian, H., Scott, A. F., Phillips, D. G., and Antonarakis, S. E. (1988). Haemophilia A resulting from de novo insertion of L1 sequences represents a novel mechanism for mutation in man. Nature 332, 164-166. doi: 10.1038/332164a0

Kemp, J. R., and Longworth, M. S. (2015). Crossing the LINE toward genomic instability: LINE-1 retrotransposition in cancer. Front. Chem. 3:68. doi: 10. 3389/fchem.2015.00068

Khalid, M., Bojang, P. Jr., Hassanin, A. A. I., Bowers, E. C., Reyes-Reyes, E. M. I, Ramos, N., et al. (2018). Line-1: Implications in the etiology of cancer, clinical applications, and pharmacologic targets. Mutat. Res. 778, 51-60. doi: 10.1016/ j.mrrev.2018.09.003

Kines, K. J., Sokolowski, M., deHaro, D. L., Christian, C. M., and Belancio, V. P. (2014). Potential for genomic instability associated with retrotranspositionallyincompetent L1 loci. Nucleic Acids Res. 42, 10488-10502. doi: 10.1093/nar/ gku687

Ko, E.-J., Oh, Y. L., Kim, H. Y., Eo, W. K., Kim, H., Ock, M. S., et al. (2019). Correlation of long interspersed element-1 open reading frame 1 and c-Met proto-oncogene protein expression in ovarian cancer. Genes Genomics 41, 1293-1299. doi: 10.1007/s13258-019-00858-y

Kosumi, K., Baba, Y., Okadome, K., Yagi, T., Kiyozumi, Y., Yoshida, N., et al. (2019). Tumor long-interspersed nucleotide element-1 methylation level and immune response to esophageal cancer. Ann. Surg. doi: 10.1097/SLA. 0000000000003264

Lai, D.-W., Liu, S.-H., Karlsson, A. I., Lee, W.-J., Wang, K.-B., Chen, Y.-C., et al. (2014). The novel Aryl hydrocarbon receptor inhibitor biseugenol inhibits gastric tumor growth and peritoneal dissemination. Oncotarget 5, 7788-7804. doi: 10.18632/oncotarget.2307

Lander, E. S., Linton, L. M., Birren, B., Nusbaum, C., Zody, M. C., Baldwin, J., et al. (2001). Initial sequencing and analysis of the human genome. Nature 409, 860-921. doi: 10.1038/35057062

Landriscina, M., Fabiano, A., Altamura, S., Bagalà, C., Piscazzi, A., Cassano, A., et al. (2005). Reverse transcriptase inhibitors down-regulate cell proliferation in vitro and in vivo and restore thyrotropin signaling and iodine uptake in human thyroid anaplastic carcinoma. J. Clin. Endocrinol. Metab. 90, 5663-5671. doi: 10.1210/jc.2005-0367

Langer, C. J., Gadgeel, S. M., Borghaei, H., Papadimitrakopoulou, V. A., Patnaik, A., Powell, S. F., et al. (2016). Carboplatin and pemetrexed with or without pembrolizumab for advanced, non-squamous non-small-cell lung cancer: a randomised, phase 2 cohort of the open-label KEYNOTE-021 study. Lancet Oncol. 17, 1497-1508. doi: 10.1016/S1470-2045(16)30498-3

Lavasanifar, A., Sharp, C. N., Korte, E. A., Yin, T., Hosseinnejad, K., and Jortani, S. A. (2019). Long interspersed nuclear element-1 mobilization as a target in cancer diagnostics, prognostics and therapeutics. Clin. Chim. Acta 493, 52-62. doi: 10.1016/j.cca.2019.02.015

Lavie, L., Maldener, E., Brouha, B., Meese, E. U., and Mayer, J. (2004). The human L1 promoter: variable transcription initiation sites and a major impact of 
upstream flanking sequence on promoter activity. Genome Res. 14, 2253-2260. doi: 10.1101/gr.2745804

Li, S., Yang, Q., Hou, Y., Jiang, T., Zong, L., Wang, Z., et al. (2018). Hypomethylation of LINE-1 elements in schizophrenia and bipolar disorder. J. Psychiatr. Res. 107, 68-72. doi: 10.1016/j.jpsychires.2018.10.009

Li, W., Jin, Y., Prazak, L., Hammell, M., and Dubnau, J. (2012). Transposable elements in TDP-43-mediated neurodegenerative disorders. PLoS One 7:e44099. doi: 10.1371/journal.pone.0044099

Lindqvist, D., Dhabhar, F. S., James, S. J., Hough, C. M., Jain, F. A., Bersani, F. S., et al. (2017). Oxidative stress, inflammation and treatment response in major depression. Psychoneuroendocrinology 76, 197-205. doi: 10.1016/j.psyneuen. 2016.11.031

Liu, S., Du, T., Liu, Z., Shen, Y., Xiu, J., and Xu, Q. (2016). Inverse changes in L1 retrotransposons between blood and brain in major depressive disorder. Sci. Rep. 6:37530. doi: 10.1038/srep37530

Lunt, S. Y., and Vander Heiden, M. G. (2011). Aerobic glycolysis: meeting the metabolic requirements of cell proliferation. Annu. Rev. Cell Dev. Biol. 27, 441-464. doi: 10.1146/annurev-cellbio-092910-154237

Mangiacasale, R., Pittoggi, C., Sciamanna, I., Careddu, A., Mattei, E., Lorenzini, R., et al. (2003). Exposure of normal and transformed cells to nevirapine, a reverse transcriptase inhibitor, reduces cell growth and promotes differentiation. Oncogene 22, 2750-2761. doi: 10.1038/sj.onc.1206354

Martin-Nunez, G. M., Rubio-Martin, E., Cabrera-Mulero, R., Rojo-Martinez, G., Olveira, G., Valdes, S., et al. (2014). Type 2 diabetes mellitus in relation to global LINE-1 DNA methylation in peripheral blood: a cohort study. Epigenetics 9, 1322-1328. doi: 10.4161/15592294.2014.969617

Mathias, S. L., Scott, A. F., Kazazian, H. H. Jr., Boeke, J. D., and Gabriel, A. (1991). Reverse transcriptase encoded by a human transposable element. Science 254, 1808-1810. doi: 10.1126/science.1722352

Mavragani, C. P., Sagalovskiy, I., Guo, Q., Nezos, A., Kapsogeorgou, E. K., Lu, P., et al. (2016). Expression of long interspersed nuclear element 1 retroelements and induction of type I interferon in patients with systemic autoimmune disease. Arthritis Rheum. 68, 2686-2696. doi: 10.1002/art.39795

Messiaen, L., Vogt, J., Bengesser, K., Fu, C., Mikhail, F., Serra, E., et al. (2011). Mosaic type-1 NF1 microdeletions as a cause of both generalized and segmental neurofibromatosis type-1 (NF1). Hum. Mutat. 32, 213-219. doi: 10.1002/humu. 21418

Miki, Y., Nishisho, I., Horii, A., Miyoshi, Y., Utsunomiya, J., Kinzler, K. W., et al. (1992). Disruption of the APC gene by a retrotransposal insertion of L1 sequence in a colon cancer. Cancer Res. 52, 643-645.

Morrish, T. A., Garcia-Perez, J. L., Stamato, T. D., Taccioli, G. E., Sekiguchi, J., and Moran, J. V. (2007). Endonuclease-independent LINE-1 retrotransposition at mammalian telomeres. Nature 446, 208-212. doi: 10.1038/nature 05560

Morrish, T. A., Gilbert, N., Myers, J. S., Vincent, B. J., Stamato, T. D., Taccioli, G. E., et al. (2002). DNA repair mediated by endonuclease-independent LINE-1 retrotransposition. Nat. Genet. 31, 159-165. doi: 10.1038/ng898

Morse, B., Rotherg, P. G., South, V. J., Spandorfer, J. M., and Astrin, S. M. (1988). Insertional mutagenesis of the myc locus by a LINE-1 sequence in a human breast carcinoma. Nature 333, 87-90. doi: 10.1038/333087a0

Muotri, A. R., Marchetto, M. C., Coufal, N. G., Oefner, R., Yeo, G., Nakashima, K., et al. (2010). L1 retrotransposition in neurons is modulated by MeCP2. Nature 468, 443-446. doi: 10.1038/nature09544

Nishikawa, Y., Nakayama, R., Obika, S., Ohsaki, E., Ueda, K., and Honda, T. (2018). Inhibition of LINE-1 retrotransposition by capsaicin. Int. J. Mol. Sci. 19, 3243. doi: $10.3390 /$ ijms 19103243

Odunsi, K., Matsuzaki, J., James, S. R., Mhawech-Fauceglia, P., Tsuji, T., Miller, A., et al. (2014). Epigenetic potentiation of NY-ESO-1 vaccine therapy in human ovarian cancer. Cancer Immunol. Res. 2, 37-49. doi: 10.1158/2326-6066.CIR13-0126

Ogino, S., Nosho, K., Kirkner, G. J., Kawasaki, T., Chan, A. T., Schernhammer, E. S., et al. (2008). A cohort study of tumoral LINE-1 hypomethylation and prognosis in colon cancer. J. Natl. Cancer Inst.100, 1734-1738. doi: 10.1093/jnci/ $\operatorname{djn} 359$

Oricchio, E., Sciamanna, I., Beraldi, R., Tolstonog, G. V., Schumann, G. G., and Spadafora, C. (2007). Distinct roles for LINE-1 and HERV-K retroelements in cell proliferation, differentiation and tumor progression. Oncogene 26, 42264233. doi: 10.1038/sj.onc. 1210214
Payer, L. M., and Burns, K. H. (2019). Transposable elements in human genetic disease. Nat. Rev. Genet. 20, 760-772. doi: 10.1038/s41576-019-0165-8

Pearce, M. S., McConnell, J. C., Potter, C., Barrett, L. M., Parker, L., Mathers, J. C., et al. (2012). Global LINE-1 DNA methylation is associated with blood glycaemic and lipid profiles. Int. J. Epidemiol. 41, 210-217. doi: 10.1093/ije/ dys020

Pedersen, I. M., and Zisoulis, D. G. (2016). Transposable elements and miRNA: regulation of genomic stability and plasticity. Mob. Genet. Elements 6:e1175537. doi: 10.1080/2159256X.2016.1175537

Powell, J. B., Goode, G. D., and Eltom, S. E. (2013). The Aryl hydrocarbon receptor: a target for breast cancer therapy. J. Cancer Ther. 4, 1177-1186. doi: 10.4236/ jct.2013.47137

Richardson, S. R., Doucet, A. J., Kopera, H. C., Moldovan, J. B., Garcia-Perez, J. L., and Moran, J. V. (2015). The influence of LINE-1 and SINE retrotransposons on mammalian genomes. Microbiol. Spectr. 3:MDNA3-2014. doi: 10.1128/ microbiolspec.MDNA3-0061-2014

Rodic, N. (2018). LINE-1 activity and regulation in cancer. Front. Biosci. 23:16801686. doi: $10.2741 / 4666$

Rodic, N., and Burns, K. H. (2013). Long interspersed element-1 (LINE-1): passenger or driver in human neoplasms? PLoS Genet 9:e1003402. doi: 10.1371/ journal.pgen.1003402

Rodic, N., Sharma, R., Sharma, R., Zampella, J., Dai, L., Taylor, M. S., et al. (2014). Long interspersed element-1 protein expression is a hallmark of many human cancers. Am. J. Pathol. 184, 1280-1286. doi: 10.1016/j.ajpath.2014. 01.007

Rodriguez-Martin, B., Alvarez, E. G., Baez-Ortega, A., Zamora, J., Supek, F., Demeulemeester, J., et al. (2020). Pan-cancer analysis of whole genomes identifies driver rearrangements promoted by LINE-1 retrotransposition. Nat. Genet. 52, 306-319. doi: 10.1038/s41588-019-0562-0

Roman-Gomez, J., Jimenez-Velasco, A., Agirre, X., Cervantes, F., Sanchez, J., Garate, L., et al. (2005). Promoter hypomethylation of the LINE-1 retrotransposable elements activates sense/antisense transcription and marks the progression of chronic myeloid leukemia. Oncogene 24, 7213-7223. doi: 10.1038/sj.onc.1208866

Rozek, L. S., Virani, S., Bellile, E. L., Taylor, J. M. G., Sartor, M. A., Zarins, K. R., et al. (2019). Soy isoflavone supplementation increases long interspersed nucleotide element-1 (LINE-1) methylation in head and neck squamous cell carcinoma. Nutr. Cancer 71, 772-780. doi: 10.1080/01635581.2019.157 7981

Saito, K., Kawakami, K., Matsumoto, I., Oda, M., Watanabe, G., and Minamoto, T. (2010). Long interspersed nuclear element 1 hypomethylation is a marker of poor prognosis in stage IA non-small cell lung cancer. Clin. Cancer Res. 16, 2418-2426. doi: 10.1158/1078-0432.CCR-09-2819

Sbardella, G., Mai, A., Bartolini, S., Castellano, S., Cirilli, R., Rotili, D., et al. (2011). Modulation of cell differentiation, proliferation, and tumor growth by dihydrobenzyloxopyrimidine non-nucleoside reverse transcriptase inhibitors. J. Med. Chem. 54, 5927-5936. doi: 10.1021/jm200734j

Schulz, W. A., Steinhoff, C., and Florl, A. R. (2006). Methylation of endogenous human retroelements in health and disease. Curr. Top. Microbiol. Immunol. 310 , 211-250. doi: 10.1007/3-540-31181-5_11

Sciamanna, I., Landriscina, M., Pittoggi, C., Quirino, M., Mearelli, C., Beraldi, R., et al. (2005). Inhibition of endogenous reverse transcriptase antagonizes human tumor growth. Oncogene 24, 3923-3931. doi: 10.1038/sj.onc.1208562

Sciamanna, I., Sinibaldi-Vallebona, P., Serafino, A., and Spadafora, C. (2018). LINE-1-encoded reverse transcriptase as a target in cancer therapy. Front. Biosci. 23:1360-1369. doi: 10.2741/4648

Shah, A. K., Saunders, N. A., Barbour, A. P., and Hill, M. M. (2013). Early diagnostic biomarkers for esophageal adenocarcinoma-the current state of play. Cancer Epidemiol. Biomark. Prevent. 22, 1185-1209. doi: 10.1158/1055-9965.EPI-121415

Shigaki, H., Baba, Y., Watanabe, M., Murata, A., Iwagami, S., Miyake, K., et al. (2013). LINE-1 hypomethylation in gastric cancer, detected by bisulfite pyrosequencing, is associated with poor prognosis. Gastric Cancer 16, 480-487. doi: 10.1007/s10120-012-0209-7

Sinibaldi-Vallebona, P., Lavia, P., Garaci, E., and Spadafora, C. (2006). A role for endogenous reverse transcriptase in tumorigenesis and as a target in differentiating cancer therapy. Genes Chrom. Cancer 45, 1-10. doi: 10.1002/ gcc. 20266 
Suarez, N. A., Macia, A., and Muotri, A. R. (2018). LINE-1 retrotransposons in healthy and diseased human brain. Dev. Neurobiol. 78, 434-455. doi: 10.1002/ dneu. 22567

Sultana, T., van Essen, D., Siol, O., Bailly-Bechet, M., Philippe, C., El Aabidine, A. Z., et al. (2019). The landscape of L1 retrotransposons in the human genome is shaped by pre-insertion sequence biases and post-insertion selection. Mol. Cell 74, 555-570.e7. doi: 10.1016/j.molcel.2019.02.036

Swergold, G. D. (1990). Identification, characterization, and cell specificity of a human LINE-1 promoter. Mol. Cell. Biol. 10, 6718-6729. doi: 10.1128/MCB. 10.12 .6718

Tang, M. L., Xiao, P., Zou, J. Z., Cao, D. D., Li, Y. Y., and Chang, H. B. (2018). [Effect of LINE1-ORF1p overexpression on the proliferation of nephroblastoma WT_CLS1 cells]. Chin. J. Contemp. Pediatr. 20, 501-507.

Taylor, J. A., Shioda, K., Mitsunaga, S., Yawata, S., Angle, B. M., Nagel, S. C., et al. (2018). Prenatal exposure to bisphenol a disrupts naturally occurring bimodal dna methylation at proximal promoter of fggy, an obesity-relevant gene encoding a carbohydrate kinase, in gonadal white adipose tissues of CD-1 mice. Endocrinology 159, 779-794. doi: 10.1210/en.2017-00711

Teerawattanapong, N., Udomsinprasert, W., Ngarmukos, S., Tanavalee, A., and Honsawek, S. (2019). Blood leukocyte LINE-1 hypomethylation and oxidative stress in knee osteoarthritis. Heliyon 5:e1774. doi: 10.1016/j.heliyon.2019. e01774

Teneng, I., Stribinskis, V., and Ramos, K. S. (2007). Context-specific regulation of LINE-1. Genes Cells 12, 1101-1110. doi: 10.1111/j.1365-2443.2007.01117.x

Tharp, M. E., Malki, S., and Bortvin, A. (2020). Maximizing the ovarian reserve in mice by evading LINE-1 genotoxicity. Nat. Commun. 11:330. doi: 10.1038/ s41467-019-14055-8

Thayer, R. E., Singer, M. F., and Fanning, T. G. (1993). Undermethylation of specific LINE-1 sequences in human cells producing a LINE-1-encoded protein. Gene 133, 273-277. doi: 10.1016/0378-1119(93)90651-I

Turcot, V., Tchernof, A., Deshaies, Y., Perusse, L., Belisle, A., Marceau, S., et al. (2012). LINE-1 methylation in visceral adipose tissue of severely obese individuals is associated with metabolic syndrome status and related phenotypes. Clin. Epigenet. 4:10. doi: 10.1186/1868-7083-4-10

Twyffels, L., Gueydan, C., and Kruys, V. (2014). Transportin-1 and Transportin-2: protein nuclear import and beyond. FEBS Lett. 588, 1857-1868. doi: 10.1016/j. febslet.2014.04.023

van den Hurk, J. A., van de Pol, D. J., Wissinger, B., van Driel, M. A., Hoefsloot, L. H. I, de Wijs, J., et al. (2003). Novel types of mutation in the choroideremia (CHM) gene: a full-length L1 insertion and an intronic mutation activating a cryptic exon. Hum. Genet. 113, 268-275. doi: 10.1007/s00439-003-0970-0

Vogt, J., Bengesser, K., Claes, K. B. M., Wimmer, K., Mautner, V.-F., van Minkelen, R., et al. (2014). SVA retrotransposon insertion-associated deletion represents a novel mutational mechanism underlying large genomic copy number changes with non-recurrent breakpoints. Genome Biol. 15, R80-R80. doi: 10.1186/gb2014-15-6-r80

Vuong, L. M., Pan, S., and Donovan, P. J. (2019). Proteome profile of endogenous retrotransposon-associated complexes in human embryonic stem cells. Proteomics 19:e1900169. doi: 10.1002/pmic.201900169

Wang, L., and Jordan, I. K. (2018). Transposable element activity, genome regulation and human health. Curr. Opin. Genet. Dev. 49, 25-33. doi: 10.1016/ j.gde.2018.02.006

Wang, L., Wang, F., Guan, J., Le, J., Wu, L., Zou, J., et al. (2010). Relation between hypomethylation of long interspersed nucleotide elements and risk of neural tube defects. Am. J. Clin. Nutr. 91, 1359-1367. doi: 10.3945/ajcn.2009.28858
Wang, X. Y., Zhang, Y., Yang, N., Cheng, H., and Sun, Y. J. (2020). DNMT3a mediates paclitaxel-induced abnormal expression of LINE-1 by increasing the intragenic methylation. Hereditas 42, 100-111.

Wanichnopparat, W., Suwanwongse, K., Pin-On, P., Aporntewan, C., and Mutirangura, A. (2013). Genes associated with the cis-regulatory functions of intragenic LINE-1 elements. BMC Genomics 14:205. doi: 10.1186/1471-216414-205

Weber, B., Kimhi, S., Howard, G., Eden, A., and Lyko, F. (2010). Demethylation of a LINE-1 antisense promoter in the cMet locus impairs Met signalling through induction of illegitimate transcription. Oncogene 29, 5775-5784. doi: 10.1038/ onc.2010.227

Wei, W., Gilbert, N., Ooi, S. L., Lawler, J. F., Ostertag, E. M., Kazazian, H. H., et al. (2001). Human L1 retrotransposition: cis preference versus trans complementation. Mol. Cell. Biol. 21, 1429-1439. doi: 10.1128/MCB.21.4.14291439.2001

Wilson, A. S., Power, B. E., and Molloy, P. L. (2007). DNA hypomethylation and human diseases. Biochim. Biophys. Acta 1775, 138-162. doi: 10.1016/j.bbcan. 2006.08.007

Wimmer, K., Callens, T., Wernstedt, A., and Messiaen, L. (2011). The NF1 gene contains hotspots for L1 endonuclease-dependent de novo insertion. PLoS Genet. 7:e1002371. doi: 10.1371/journal.pgen.1002371

Xiang, Y., Yan, K., Zheng, Q., Ke, H., Cheng, J., Xiong, W., et al. (2019). Histone demethylase KDM4B promotes DNA damage by activating long interspersed nuclear element-1. Cancer Res. 79, 86-98. doi: 10.1158/0008-5472.CAN-181310

Yooyongsatit, S., Ruchusatsawat, K., Noppakun, N., Hirankarn, N., Mutirangura, A., and Wongpiyabovorn, J. (2015). Patterns and functional roles of LINE-1 and Alu methylation in the keratinocyte from patients with psoriasis vulgaris. J. Hum. Genet. 60, 349-355. doi: 10.1038/jhg.2015.33

Zhang, R., Zhang, F., Sun, Z., Liu, P., Zhang, X., Ye, Y., et al. (2019). LINE1 retrotransposition promotes the development and progression of lung squamous cell carcinoma by disrupting the tumor-suppressor gene FGGY. Cancer Res. 79, 4453-4465. doi: 10.1158/0008-5472.CAN-19-0076

Zhang, Y., Zagnitko, O., Rodionova, I., Osterman, A., and Godzik, A. (2011). The FGGY carbohydrate kinase family: insights into the evolution of functional specificities. PLoS Comput. Biol. 7:e1002318. doi: 10.1371/journal.pcbi.100 2318

Zhao, K., Du, J., Peng, Y., Li, P., Wang, S., Wang, Y., et al. (2018). LINE1 contributes to autoimmunity through both RIG-I- and MDA5-mediated RNA sensing pathways. J. Autoimmun. 90, 105-115. doi: 10.1016/j.jaut.2018. 02.007

Zhu, J., Ling, Y., Xu, Y., Lu, M.-Z., Liu, Y.-P., and Zhang, C.-S. (2015). Elevated expression of MDR1 associated with Line-1 hypomethylation in esophageal squamous cell carcinoma. Int. J. Clin. Exp. Pathol. 8, 14392-14400.

Conflict of Interest: The authors declare that the research was conducted in the absence of any commercial or financial relationships that could be construed as a potential conflict of interest.

Copyright (c) 2020 Zhang, Zhang and Yu. This is an open-access article distributed under the terms of the Creative Commons Attribution License (CC BY). The use, distribution or reproduction in other forums is permitted, provided the original author(s) and the copyright owner(s) are credited and that the original publication in this journal is cited, in accordance with accepted academic practice. No use, distribution or reproduction is permitted which does not comply with these terms. 